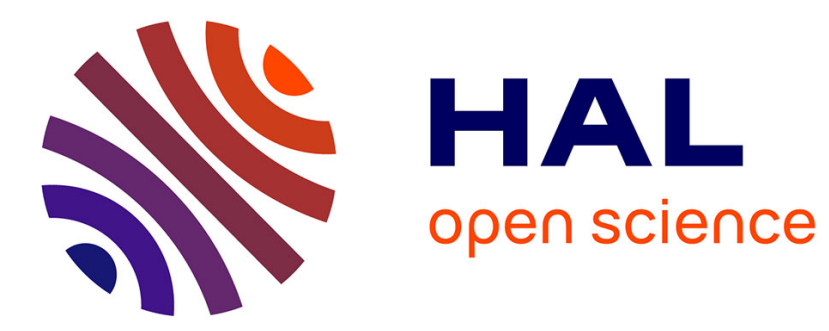

\title{
Sensitivity of the Solution Set to Second Order Evolution Inclusions
}

Jiangfeng Han, Stanislaw Migorski

\section{To cite this version:}

Jiangfeng Han, Stanislaw Migorski. Sensitivity of the Solution Set to Second Order Evolution Inclusions. 26th Conference on System Modeling and Optimization (CSMO), Sep 2013, Klagenfurt, Austria. pp.138-147, 10.1007/978-3-662-45504-3_13 . hal-01286406

\section{HAL Id: hal-01286406 https://hal.inria.fr/hal-01286406}

Submitted on 10 Mar 2016

HAL is a multi-disciplinary open access archive for the deposit and dissemination of scientific research documents, whether they are published or not. The documents may come from teaching and research institutions in France or abroad, or from public or private research centers.
L'archive ouverte pluridisciplinaire HAL, est destinée au dépôt et à la diffusion de documents scientifiques de niveau recherche, publiés ou non, émanant des établissements d'enseignement et de recherche français ou étrangers, des laboratoires publics ou privés. 


\title{
Sensitivity of the solution set to second order evolution inclusions ${ }^{\star}$
}

\author{
Jiangfeng Han and Stanislaw Migorski \\ Faculty of Mathematics and Computer Science \\ Jagiellonian University, Institute of Computer Science \\ ul. Łojasiewicza 6, 30348 Krakow, Poland
}

\begin{abstract}
In this note we study second order evolution inclusions in the framework of evolution triple of spaces. The existence of mild solutions (i.e. trajectory-selection pairs) to the inclusion, and the upper and lower semicontinuity properties of the solution set with respect to a parameter are established.
\end{abstract}

Keywords: evolution inclusion, Kuratowski convergence, upper semicontinuity, lower semicontinuity.

\section{Introduction and preliminaries}

In this paper we investigate a class of systems described by abstract second order evolution equations with multivalued right hand side. We consider Problem $(\mathrm{P})$ of the form

$$
\left\{\begin{array}{l}
\ddot{x}(t)+A(t, \dot{x}(t))+B x(t) \in F(t, x(t), \dot{x}(t)) \text { a.e. } t \in(0, T), \\
x(0)=x_{0}, \quad \dot{x}(0)=x_{1}
\end{array}\right.
$$

and the following sequence of Problems $(\mathrm{P})_{\mathrm{n}}, n \in \mathbb{N}$, that can be regarded as the perturbed ones

$$
\left\{\begin{array}{l}
\ddot{x}(t)+A_{n}(t, \dot{x}(t))+B_{n} x(t) \in F_{n}(t, x(t), \dot{x}(t)) \text { a.e. } t \in(0, T), \\
x(0)=x_{0}^{n}, \quad \dot{x}(0)=x_{1}^{n}
\end{array}\right.
$$

The goal is to establish the lower and upper semicontinuity properties of the solution set to Problem (P) with respect to the parameter $n \in \mathbb{N}$. The main result concerns the Kuratowski convergence of the sequence of solution sets to

\footnotetext{
* This research was supported by the Marie Curie International Research Staff Exchange Scheme Fellowship within the 7th European Community Framework Programme under Grant Agreement No. 295118, the National Science Center of Poland under grant no. N N201 604640, the International Project co-financed by the Ministry of Science and Higher Education of Republic of Poland under grant no. W111/7.PR/2012, the National Science Center of Poland under Maestro Advanced Project no. DEC-2012/06/A/ST1/00262.
} 
Problem $(\mathrm{P})_{\mathrm{n}}$ to that of Problem $(\mathrm{P})$. Evolution inclusions of second order and their applications have been considered in several papers, see e.g. [6-9] and the references therein.

We introduce below the notation and preliminary material needed in the next sections. For a Banach space $X$, we indicate by $w-X, s-X$ the space $X$ equipped with the weak and the strong (norm) topology, respectively. Let $(\Omega, \Sigma, \mu)$ be a measure space. A multifunction $F$ defined on $\Omega$ with values in the space $2^{X}$ of all nonempty subsets of $X$ is called measurable if $F^{-}(E)=\{\omega \in \Omega \mid$ $F(\omega) \cap E \neq \emptyset\} \in \Sigma$ for every closed set $E \subset X$. It is called graph measurable if $\operatorname{Gr} F=\{(\omega, x) \in \Omega \times X \mid x \in F(\omega)\} \in \Sigma \times \mathcal{B}(X)$ where $\mathcal{B}(X)$ is the family of all Borel subsets of $X$. We denote by $S_{F}^{r}, 1 \leq r \leq \infty$, the set of all selectors of $F$ that belong to $L^{r}(\Omega ; X)$, i.e., $S_{F}^{r}=\left\{f \in L^{r}(\Omega ; X) \mid f(\omega) \in\right.$ $F(\omega) \mu$ a.e. $\}$. The symbol $\mathcal{P}_{f(c)}(X)$ stands for the family of all closed, (convex) subsets of $2^{X}$. On $\mathcal{P}_{f}(X)$ we define the Hausdorff metric, by setting $h(A, B)=$ $\max \left\{\sup _{a \in A} d(a, B), \sup _{b \in B} d(b, A)\right\}$. We also write $|A|=\sup \{|a| \mid a \in A\}$.

Given $\left\{S_{n}, S\right\}_{n \in \mathbb{N}} \subset 2^{Z}$, we define (see e.g. [3]) the sequential Kuratowski lower and upper limits respectively by $\tau_{Z^{-}} \lim \inf S_{n}=\left\{z \in Z \mid \exists z_{n} \in S_{n}, z_{n} \rightarrow\right.$ $z$ in $\tau_{Z^{-}} Z$, as $\left.n \rightarrow+\infty\right\}$ and $\tau_{Z^{-}} \lim \sup S_{n}=\left\{z \in Z \mid \exists\left\{n_{\nu}\right\}, z_{n_{\nu}} \in S_{n_{\nu}}, z_{n_{\nu}} \rightarrow\right.$ $z$ in $\tau_{Z}-Z$, as $\left.\nu \rightarrow+\infty\right\}$. We say that $S_{n}$ converge to $S$ in the Kuratowski sense (denoted by $S_{n} \stackrel{K}{\longrightarrow} S$ ) if and only if $\tau_{Z^{-}} \lim \sup S_{n} \subset S \subset \tau_{Z^{-}} \lim \inf S_{n}$.

Let $\left(Y, \tau_{Y}\right)$ and $\left(Z, \tau_{Z}\right)$ be Hausdorff topological spaces. A multifunction $G: Y \rightarrow 2^{Z}$ is said to be $\left(\tau_{Y}-\tau_{Z}\right)$ upper semicontinuous (usc) (respectively lower semicontinuous (lsc)) (cf. [2], Section 4.7 of [3]), if for every $C \subset Z$ closed in $\tau_{Z}$ topology, $G^{-}(C)$ (respectively, $G^{+}(C)=\{y \in Y \mid G(y) \subset C\}$ ) is closed in $\tau_{Y}$ topology in $Y$. The definition of lsc is equivalent to saying that if $y_{n} \rightarrow y$ in $\tau_{Y}-Y$, then $G(y) \subset \tau_{Z}-\liminf G\left(y_{n}\right)$. For a sequence of multifunctions $G$, $G_{n}: Y \rightarrow 2^{Z}$, we write

$$
K\left(\tau_{Y}, \tau_{Z}\right) \limsup _{n \rightarrow+\infty, y \rightarrow \tilde{y}} G_{n}(y) \subset G(\widetilde{y})
$$

if $\tau_{Z^{-}} \lim \sup G_{n}\left(y_{n}\right) \subset G(y)$ for every $y_{n} \rightarrow y$ in $\tau_{Y}-Y$. Similar notation is used for $\tau_{Z}-\liminf$.

Let $H$ be a separable Hilbert space and $V$ be a reflexive Banach space which is densely, continuously and compactly embedded in $H$. Identifying $H$ with its dual $H^{*}$, we have the Gelfand triple $V \subset H \subset V^{*}$, where $V^{*}$ is the dual of $V$. Let $\langle\cdot, \cdot\rangle$ be the duality of $V$ and $V^{*}$ as well as the inner product on $H$, let $\|\cdot\|,|\cdot|$ and $\|\cdot\|_{V^{*}}$ denote the norms in $V, H$ and $V^{*}$, respectively. For $T>0$ and $2 \leq p<+\infty$, we introduce the following spaces $\mathcal{V}=L^{p}(0, T ; V)$, $\mathcal{H}=L^{p}(0, T ; H), \mathcal{H}^{*}=L^{q}(0, T ; H), \mathcal{V}^{*}=L^{q}\left(0, T ; V^{*}\right)$, where $1 / p+1 / q=1$, $1<q \leq 2$, and $\mathcal{W}=\left\{w \in \mathcal{V} \mid w^{\prime} \in \mathcal{V}^{*}\right\}$. The derivative is understood in the sense of vector valued distributions. Clearly $\mathcal{W} \subset \mathcal{V} \subset \mathcal{H} \subset \mathcal{H}^{*} \subset \mathcal{V}^{*}$. The pairing of $\mathcal{V}$ and $\mathcal{V}^{*}$ and the duality between $\mathcal{H}$ and $\mathcal{H}^{*}$ are denoted by $\langle\langle f, v\rangle\rangle=\int_{0}^{T}\langle f(s), v(s)\rangle d s$. It is well known that the embedding $\mathcal{W} \subset C(0, T ; H)$ is continuous. Since $V \subset H$ compactly we know that the embedding $\mathcal{W} \subset \mathcal{H}$ is also compact. Finally, the class of linear bounded operators from $V$ into $V^{*}$ is denoted by $\mathcal{L}\left(V, V^{*}\right)$. For additional details on the material, we refer to $[3,11]$. 


\section{Results on evolution equations}

In this section we investigate the existence, uniqueness and continuous dependence of solutions on the data for an evolution equation of second order. We consider the following problem

$$
\left\{\begin{array}{l}
\ddot{x}(t)+A(t, \dot{x}(t))+B x(t)=f(t) \text { a.e. } t \in(0, T), \\
x(0)=x_{0}, \quad \dot{x}(0)=x_{1}
\end{array}\right.
$$

A function $x \in C(0, T ; V)$ is called a solution to the problem $(E)$ if and only if $\dot{x} \in \mathcal{W}$ and $(E)$ is satisfied.

We will need the following hypotheses.

$H(A): \quad A:(0, T) \times V \rightarrow V^{*}$ is an operator such that

(1) $t \mapsto A(t, v)$ is measurable, for every $v \in V$,

(2) $v \mapsto A(t, v)$ is monotone and hemicontinuous, a.e. $t \in(0, T)$,

(3) $\langle A(t, v), v\rangle \geq c\|v\|^{p}-d|v|^{2}$ a.e. for all $v \in V$ with $c>0$ and $d \geq 0$,

(4) $\|A(t, v)\|_{V^{*}} \leq a(t)+b\|v\|^{p-1}$ for all $v \in V$, a.e. $t \in(0, T)$ with $a \in L_{+}^{q}(0, T)$ and $b>0$.

$H(B): \quad B \in \mathcal{L}\left(V, V^{*}\right)$ is symmetric and coercive (i.e., $\langle B v, v\rangle \geq m\|v\|^{2}$ for all $v \in V$ with $m>0)$.

$\underline{\left(H_{0}\right)}: \quad x_{0} \in V, x_{1} \in H$.

The proof of the following result follows from the standard application of the Galerkin method and can be found in $[1,5,6]$.

Proposition 1. Under hypotheses $H(A), H(B),\left(H_{0}\right)$ and $f \in \mathcal{H}^{*}$, the problem (E) admits a unique solution which satisfies $x \in C(0, T ; V), \dot{x} \in \mathcal{W}$, and the following estimate

$$
\|x(t)\|^{2}+|\dot{x}(t)|^{2}+\|\dot{x}\|_{\mathcal{W}}^{2} \leq C\left(1+\left\|x_{0}\right\|^{2}+\left|x_{1}\right|^{2}+\|B\|_{\mathcal{L}\left(V, V^{*}\right)}^{2}+\|f\|_{\mathcal{H}^{*}}^{q}\right)
$$

for all $t \in[0, T]$ with $C>0$.

We present now a result on the continuous dependence of solutions to the problem

$(\mathrm{E})_{n} \quad\left\{\begin{array}{l}\ddot{x}(t)+A_{n}(t, \dot{x}(t))+B_{n} x(t)=f_{n}(t) \quad \text { a.e. } t \in(0, T), \\ x(0)=x_{0}^{n}, \quad \dot{x}(0)=x_{1}^{n} .\end{array}\right.$

on the data. We will need the following assumptions.

$\underline{H(A)_{1}}: \quad A:(0, T) \times V \rightarrow V^{*}$ is such that $H(A)$ holds, $A_{n}:(0, T) \times V \rightarrow V^{*}$ satisfy $H(A)(1)(2)(3)$ uniformly with respect to $n \in \mathbb{N}$ and the condition 
$\left\|A_{n}(t, v)\right\|_{V^{*}} \leq a_{n}(t)+b\|v\|^{p-1} \quad$ for all $v \in V$, a.e. $t \in(0, T)$ with $a_{n} \in L_{+}^{q}(0, T), \sup _{n \in \mathbb{N}}\left\|a_{n}\right\|_{L^{q}}<+\infty, b>0$ and $A_{n}(\cdot, w(\cdot)) \rightarrow A(\cdot, w(\cdot))$ in $s^{-} \mathcal{V}^{*}$ for all $w \in \mathcal{V} \cap L^{\infty}(0, T ; H)$.

$H(B)_{1}: \quad B_{n} \in \mathcal{L}\left(V, V^{*}\right)$ satisfy $H(B)$ uniformly with respect to $n \in \mathbb{N}$ and $B_{n} \rightarrow B$ in $\mathcal{L}\left(V, V^{*}\right)$.

$\underline{\left(H_{0}\right)_{1}}: \quad x_{0}^{n}, x_{0} \in V, x_{1}^{n}, x_{1} \in H, x_{0}^{n} \rightarrow x_{0}$ in $s-V$ and $x_{1}^{n} \rightarrow x_{1}$ in $s-H$.

For every $n \in \mathbb{N}$, let $x_{n}$ be a solution of the problem $(\mathrm{E})_{n}$ and let $x$ be a solution of the problem $(E)$. We have

Proposition 2. If hypotheses $H(A)_{1}, H(B)_{1},\left(H_{0}\right)_{1}$ hold, $f_{n} \in \mathcal{H}^{*}, f_{n} \rightarrow f$ weakly in $\mathcal{H}^{*}$, then the sequence $\left\{\left(x_{n}, \dot{x}_{n}\right)\right\}$ converges to $(x, \dot{x})$ in $C(0, T ; V \times H)$, as $n \rightarrow+\infty$.

Proof. By Proposition 1 we know that, for every $n \in \mathbb{N}$, the problem $(\mathrm{E})_{n}$ has the unique solution $x_{n} \in C(0, T ; V)$ such that $\dot{x}_{n} \in \mathcal{W}$. From $(\mathrm{E})_{n}$ and $(E)$, we have

$$
\begin{aligned}
& \left\langle\ddot{x}_{n}(s)-\ddot{x}(s), \dot{x}_{n}(s)-\dot{x}(s)\right\rangle+\left\langle A_{n}\left(s, \dot{x}_{n}(s)\right)-A(s, \dot{x}(s)), \dot{x}_{n}(s)-\dot{x}(s)\right\rangle+ \\
& \quad+\left\langle B_{n} x_{n}(s)-B x(s), \dot{x}_{n}(s)-\dot{x}(s)\right\rangle=\left\langle f_{n}(s)-f(s), \dot{x}_{n}(s)-\dot{x}(s)\right\rangle \text { a.e. }
\end{aligned}
$$

for every $n \in \mathbb{N}$. Integrating this equality and using the monotonicity of $A_{n}(s, \cdot)$, we get

$$
\begin{aligned}
& \left|\dot{x}_{n}(t)-\dot{x}(t)\right|^{2}-\left|\dot{x}_{n}(0)-\dot{x}(0)\right|^{2}+2 \int_{0}^{t}\left\langle A_{n}(s, \dot{x}(s))-A(s, \dot{x}(s)), \dot{x}_{n}(s)-\dot{x}(s)\right\rangle d s+ \\
& +2 \int_{0}^{t}\left\langle B_{n} x_{n}(s)-B x_{n}(s), \dot{x}_{n}(s)-\dot{x}(s)\right\rangle d s+\left\langle B x_{n}(t)-B x(t), x_{n}(t)-x(t)\right\rangle- \\
& \quad-\left\langle B x_{n}(0)-B x(0), x_{n}(0)-x(0)\right\rangle \leq 2 \int_{0}^{t}\left\langle f_{n}(s)-f(s), \dot{x}_{n}(s)-\dot{x}(s)\right\rangle d s
\end{aligned}
$$

for all $t \in[0, T]$. Hence using $H(B)_{1}$ and applying the Hölder inequality, we obtain

$$
\begin{gathered}
\left|\dot{x}_{n}(t)-\dot{x}(t)\right|^{2}+m\left\|x_{n}(t)-x(t)\right\|^{2} \leq\|B\|\left\|x_{0}^{n}-x_{0}\right\|+\left|x_{1}^{n}-x_{1}\right|^{2}+ \\
+2\left\|\widehat{A}_{n}(\dot{x})-\widehat{A}(\dot{x})\right\|_{\mathcal{V}^{*}}\left\|\dot{x}_{n}-\dot{x}\right\|_{\mathcal{V}}+\widetilde{C}\left\|B_{n}-B\right\|\left\|x_{n}\right\|_{\mathcal{V}}\left\|\dot{x}_{n}-\dot{x}\right\|_{\mathcal{V}}+ \\
+2\left\langle\left\langle f_{n}-f, \dot{x}_{n}-\dot{x}\right\rangle\right\rangle
\end{gathered}
$$

for all $t \in[0, T]$, where $\widehat{A}_{n}$ and $\widehat{A}$ are the Nemitsky operators corresponding to $A_{n}$ and $A$, respectively, and $\widetilde{C}$ is a positive constant independent of $n$. On the other hand, due to $H(A)_{1}, H(B)_{1}$ and $\left(H_{0}\right)_{1}$, from Proposition 1, we have

$$
\left\|x_{n}(t)\right\|^{2}+\left|\dot{x}_{n}(t)\right|^{2}+\left\|\dot{x}_{n}\right\|_{\mathcal{W}}^{2} \leq C\left(1+\left\|x_{0}^{n}\right\|^{2}+\left|x_{1}^{n}\right|^{2}+\left\|B_{n}\right\|^{2}+\left\|f_{n}\right\|_{\mathcal{H}^{*}}^{q}\right) .
$$


Hence, it follows that $\left\{\dot{x}_{n}\right\}$ lies in a bounded subset of $\mathcal{W}$. Thus, up to a subsequence, $\dot{x}_{n}$ converges weakly in $\mathcal{W}$ and (since $\mathcal{W} \subset \mathcal{H}$ compactly) strongly in $\mathcal{H}$. So we have

$$
\lim _{n \rightarrow+\infty}\left\langle\left\langle f_{n}-f, \dot{x}_{n}-\dot{x}\right\rangle\right\rangle=0 .
$$

Using the assumptions, (2) and (3), from (1), we get $\left(x_{n}(t), \dot{x}_{n}(t)\right) \rightarrow(x(t), \dot{x}(t))$ in $s^{-}(V \times H)$ for all $t \in[0, T]$, as $n \rightarrow+\infty$. Since the solution to $(E)$ is unique, we deduce that the whole sequence $\left\{\left(x_{n}, \dot{x}_{n}\right)\right\}$ converges to $(x, \dot{x})$ in $C(0, T ; V \times H)$. The proof is completed.

In the sequel, we make use of the solution map $r: \mathcal{H}^{*} \rightarrow C(0, T ; V) \times \mathcal{W}$ for $(E)$ defined by $r(f)=(x, \dot{x})$, where $x$ (respectively $\dot{x}$ ) is the solution (and its derivative, respectively) to $(E)$. By Proposition 1 this map is well defined and Proposition 2 implies the following result.

Corollary 1. Under hypotheses $H(A), H(B)$ and $\left(H_{0}\right)$, the solution map $r$ for the problem $(E)$ is continuous from $w-\mathcal{H}^{*}$ into $C(0, T ; V \times H)$.

\section{Existence result for inclusions}

In this section we study the existence of solutions to Problem $(\mathrm{P})$. We start with the following

Definition 1. A couple $(x, f) \in C(0, T ; V) \times \mathcal{H}^{*}$ is called a mild solution to Problem $(\mathrm{P})$ if and only if $x$ is a solution to the evolution equation $(E)$ and $f(\cdot) \in S_{F(\cdot, x(\cdot), \dot{x}(\cdot))}^{q} \cdot$

Prior to the existence theorem, we state the a priori bound on the solution to the evolution inclusion. We need the following hypotheses.

$H(F): \quad F:(0, T) \times H \times H \rightarrow \mathcal{P}_{f c}(H)$ is a multifunction such that

(1) $F$ is graph measurable,

(2) $\operatorname{Gr} F(t, \cdot, \cdot)$ is sequentially closed in $H \times H \times(w-H)$, a.e. $t \in(0, T)$,

(3) $|F(t, x, y)| \leq a_{1}(t)+b_{1}|x|^{2 / q}+c_{1}|y|^{2 / q}$, a.e. $t \in(0, T)$, where $a_{1} \in L_{+}^{q}(0, T)$ and $b_{1}, c_{1}>0$.

Lemma 1. Assume $H(A), H(B), H(F)$ and $\left(H_{0}\right)$. If $(x, f)$ is a mild solution to Problem $(\mathrm{P})$, then $(x, \dot{x}, f)$ lies in a bounded set of $\left(L^{\infty}(0, T ; V) \cap W^{1, \infty}(0, T ; H)\right) \times$ $\mathcal{W} \times \mathcal{H}^{*}$.

In the proof of the next result, we follow methods used in $[4,10]$.

Theorem 1. If hypotheses $H(A), H(B), H(F)$ and $\left(H_{0}\right)$ hold, then Problem (P) admits a mild solution. 
Proof. From Lemma 1, it is clear that every solution to Problem (P) satisfies

$$
|x(t)| \leq M_{1}, \quad|\dot{x}(t)| \leq M_{2}
$$

for all $t \in(0, T)$ with positive constants $M_{1}, M_{2}$. We define multifunction $\widehat{F}:(0, T) \times H \times H \rightarrow \mathcal{P}_{f c}(H)$ by $\widehat{F}(t, x, y)=F(t, p(x, y))$, where the map $p: H \times H \rightarrow B\left(0, M_{1}\right) \times B\left(0, M_{2}\right)$ is as follows

$$
p(x, y)= \begin{cases}(x, y) & \text { if }|x| \leq M_{1} \text { and }|y| \leq M_{2}, \\ \left(\left(M_{1} x /|x|\right),\left(M_{2} y /|y|\right)\right) & \text { if }|x|>M_{1} \text { and }|y|>M_{2}, \\ \left(\left(M_{1} x /|x|\right), y\right) & \text { if }|x|>M_{1} \text { and }|y| \leq M_{2}, \\ \left(x,\left(M_{2} y /|y|\right)\right) & \text { if }|x| \leq M_{1} \text { and }|y|>M_{2} .\end{cases}
$$

Since the map $p$ is Lipschitz continuous, from the properties of $F$, we deduce that $\widehat{F}$ satisfies $H(F)(1)(2)$. Furthermore, we note that $|\widehat{F}(t, x, y)| \leq \widetilde{a}_{1}(t)$ a.e. $t \in(0, T)$, where $\widetilde{a}_{1} \in L_{+}^{q}(0, T)$ is given by $\widetilde{a}_{1}(t)=a_{1}(t)+b_{1} M_{1}^{2 / q}+c_{1} M_{2}^{2 / q}$.

We define $\mathcal{Z}=\left\{f \in \mathcal{H}^{*}|| f(t) \mid \leq \widetilde{a}_{1}(t)\right.$ a.e. $\left.t \in(0, T)\right\}$ and a multifunction $\mathcal{R}$ on $\mathcal{Z}$ by

$$
\mathcal{R}(f)=S_{\widehat{F}(\cdot, r(f)(\cdot))}^{1}=\left\{f \in L^{1}(0, T ; H) \mid f(t) \in \widehat{F}(t, r(f)(t)) \text { a.e. } t \in(0, T)\right\}
$$

(recall that $r(\cdot)$ is the solution map for the equation $(E)$ ). Since $\widehat{F}$ is graph measurable and $L^{1}$ integrably bounded, using the Aumann selection theorem (see Theorem 4.3.7 of [3]), we have $\mathcal{R}(f) \neq \emptyset$ for $f \in \mathcal{Z}$. Moreover, because $\widehat{F}$ is $\mathcal{P}_{f c}(H)$-valued and $|\widehat{F}(t, r(f)(t))| \leq \widetilde{a}_{1}(t)$ a.e. $t \in(0, T)$, we obtain that $\mathcal{R}: \mathcal{Z} \rightarrow \mathcal{P}_{f c}(\mathcal{Z})$.

We will show that $\mathcal{R}$ is $\left(\mathrm{w}-\mathcal{H}^{*}\right) \times\left(\mathrm{w}-\mathcal{H}^{*}\right)$ usc on $\mathcal{Z}$. Since $\mathcal{Z}$ is compact in $w-\mathcal{H}^{*}$, it suffices to prove (see Chapter I of [2], Section 4.1 of [3]) that $G r \mathcal{R}$ is weakly-weakly closed in $\mathcal{Z} \times \mathcal{Z}$. Let $\left(f_{n}, z_{n}\right) \in G r \mathcal{R}, f_{n} \rightarrow f$ and $z_{n} \rightarrow z$ both in $w-\mathcal{H}^{*}$. By Corollary 1 , we know that $r\left(f_{n}\right)(t) \rightarrow r(f)(t)$ in $(s-H) \times(s-H)$ for all $t \in[0, T]$. Since $\widehat{F}$ satisfies $H(F)(1)(2)$, we deduce that $w-\lim \sup \widehat{F}\left(t, r\left(f_{n}\right)(t)\right) \subset \widehat{F}(t, r(f)(t))$ a.e. $t \in(0, T)$. Using Theorem 4.7.51 of [3], we obtain

$$
\begin{aligned}
& w-\lim \sup \mathcal{R}\left(f_{n}\right)=w-\lim \sup S_{\widehat{F}\left(\cdot, r\left(f_{n}\right)(\cdot)\right)}^{1} \subset \\
& \subset S_{w-\limsup \widehat{F}\left(\cdot, r\left(f_{n}\right)(\cdot)\right)}^{1} \subset S_{\widehat{F}(\cdot, r(f)(\cdot))}^{1}=\mathcal{R}(f) .
\end{aligned}
$$

From these inclusions we have $(f, z) \in G r \mathcal{R}$. This means that $G r \mathcal{R}$ is closed in $(w-\mathcal{Z}) \times(w-\mathcal{Z})$ and proves that $\mathcal{R}$ is weakly-weakly usc on $\mathcal{Z}$.

We apply the well known Kakutani-KyFan fixed point theorem for set-valued mappings (see Chapter I.12 of [2]) to the multifunction $\mathcal{R}$. We deduce that there exists $f^{*} \in \mathcal{Z}$ such that $f^{*} \in \mathcal{R}\left(f^{*}\right)$. The corresponding pair $\left(x^{*}, \dot{x}^{*}\right)=r\left(f^{*}\right)$ is a solution to Problem $(\mathrm{P})$ with $F$ replaced by $\widehat{F}$. However, the same estimates as in Lemma 1 (cf. also (4)), imply that $\left|x^{*}(t)\right| \leq M_{1},\left|\dot{x}^{*}(t)\right| \leq M_{2}$ for every 
$t \in(0, T)$. Thus $\widehat{F}\left(t, x^{*}(t), \dot{x}^{*}(t)\right)=F\left(t, x^{*}(t), \dot{x}^{*}(t)\right)$ for a.e. $t \in(0, T)$, which means that $\left(x^{*}, f^{*}\right)$ is a mild solution to Problem $(\mathrm{P})$. This completes the proof of the theorem.

Corollary 2. If $F(t, u, v)=\{f(t, u, v)\}$, where $f:(0, T) \times H \times H \rightarrow H$ is a function measurable in $t$, continuous in $(u, v)$ and

$$
|f(t, u, v)| \leq a_{1}(t)+b_{1}|u|^{2 / q}+c_{1}|v|^{2 / q} \text { a.e. } t \in(0, T)
$$

for all $u, v \in H$, then Theorem 1 ensures that the Cauchy problem for the nonlinear equation $\ddot{x}(t)+A(t, \dot{x}(t))+B x(t)=f(t, x(t), \dot{x}(t))$ has at least one solution.

Let $S$ be the set of mild solutions to Problem (P) and let

$$
\mathcal{M}=\left\{(x, \dot{x}, f) \in C(0, T ; V \times H) \times \mathcal{H}^{*} \mid(x, f) \in S\right\} .
$$

Corollary 3. Under the hypotheses of Theorem 1, the set $\mathcal{M}$ is nonempty, compact subset of $C(0, T ; V \times H) \times\left(w-\mathcal{H}^{*}\right)$.

Proof. The nonemptiness of $\mathcal{M}$ follows from Theorem 1. Let $\left\{\left(x_{k}, \dot{x}_{k}, f_{k}\right)\right\}_{k \in \mathbb{N}}$ $\subset \mathcal{M}$. We will show that this sequence has a subsequence which converges in an appropriate topology to an element of $\mathcal{M}$. By the definition, $x_{k}$ satisfies the evolution equation $(E)$ with the right-hand side $f_{k}$ and $f_{k}(\cdot) \in S_{F\left(\cdot, x_{k}(\cdot), \dot{x}_{k}(\cdot)\right)}^{q}$. From Lemma 1, we obtain in particular that $f_{k}$ remains in a bounded subset of $\mathcal{H}^{*}$. So after a possible passing to subsequence, we have $f_{k} \rightarrow f$ weakly in $\mathcal{H}^{*}$, as $k \rightarrow+\infty$, with $f \in \mathcal{H}^{*}$. Corollary 1 says that $r\left(f_{k}\right) \rightarrow r(f)$ in $C(0, T ; V \times H)$, where $r(f)=(x, \dot{x})$ is a solution to $(E)$. In order to conclude the proof, it suffices to show that $f$ is a selection for $F(\cdot, x(\cdot), \dot{x}(\cdot))$. From Theorem 4.7.44 of [3], we have

$$
f(t) \in \overline{c o} w-\limsup \left\{f_{k}(t)\right\}_{k \geq 1} \subset \overline{c o} w-\lim \sup F\left(t, x_{k}(t), \dot{x}_{k}(t)\right)
$$

a.e. $t \in(0, T)$. Since $\left(x_{k}(t), \dot{x}_{k}(t)\right) \rightarrow(x(t), \dot{x}(t))$ in $s^{-}(H \times H)$ for all $t \in$ $[0, T]$, from $H(F)(1)(2)$, we easily deduce that $w$ - limsup $F\left(t, x_{k}(t), \dot{x}_{k}(t)\right) \subset$ $F(t, x(t), \dot{x}(t))$ a.e. $t \in(0, T)$. Hence, we get $f(t) \in F(t, x(t), \dot{x}(t))$ a.e. $t \in(0, T)$. So we have obtained $(x, \dot{x}, f) \in \mathcal{M}$ which completes the proof.

\section{Upper semicontinuity property of the solution set}

Consider now a sequence of evolution inclusions Problem $(\mathrm{P})_{\mathrm{n}}$. Let us denote by $S_{n}$ the set of mild solutions to Problem $(\mathrm{P})_{\mathrm{n}}$, i.e., $S_{n}=\left\{(x, f) \in C(0, T ; V) \times \mathcal{H}^{*} \mid\right.$ $(x, f)$ is a mild solution to Problem $\left.(\mathrm{P})_{\mathrm{n}}\right\}$.

Theorem 2. Suppose that hypotheses $H(A)_{1}, H(B)_{1},\left(H_{0}\right)_{1}$ hold, $F, F_{n}:(0, T)$ $\times H \times H \rightarrow \mathcal{P}_{f c}(H)$ are multifunctions satisfying $H(F)$ uniformly with respect to $n \in \mathbb{N}$ and

$$
\begin{gathered}
K(s-(H \times H) \times(w-H)) \limsup _{n \rightarrow+\infty,(u, v) \rightarrow(\tilde{u}, \tilde{v})} F_{n}(t, u, v) \subset F(t, \tilde{u}, \tilde{v}) \text { a.e. } \\
\text { If }\left(x_{n}, f_{n}\right) \in S_{n}, n \in \mathbb{N} \text { and } f_{n} \rightarrow f \text { in } w-\mathcal{H}^{*} \text {, then }(x, f) \in S .
\end{gathered}
$$


Proof. From Theorem 1 we know that $S_{n}, S \neq \emptyset$. Let $\left(x_{n}, f_{n}\right) \in S_{n}$ for $n \in \mathbb{N}$ and $f_{n} \rightarrow f$ weakly in $\mathcal{H}^{*}$. By Proposition 2 , we infer that $\left(x_{n}, \dot{x}_{n}\right)$ converges in $C(0, T ; V \times H)$ to $(x, \dot{x})$, as $n \rightarrow+\infty$, where $x$ is a solution to the equation $(E)$ (corresponding to the right hand side $f$ ). It remains to prove that $f(\cdot) \in$ $S_{F(\cdot, x(\cdot), \dot{x}(\cdot))}^{q}$. From Theorem 4.7.44 of [3], we have

$$
f(t) \in \overline{c o} w-\lim \sup \left\{f_{n}(t)\right\}_{n \in \mathbb{N}} \subset \overline{c o} w-\lim \sup F_{n}\left(t, x_{n}(t), \dot{x}_{n}(t)\right)
$$

a.e. $t \in(0, T)$ and by (6) we obtain

$$
w-\limsup F_{n}\left(t, x_{n}(t), \dot{x}_{n}(t)\right) \subset F(t, x(t), \dot{x}(t)) \text { a.e. } t \in(0, T) .
$$

This facts imply $f(t) \in F(t, x(t), \dot{x}(t))$ a.e. $t \in(0, T)$. Hence $(x, f)$ is a mild solution to Problem (P) which concludes the proof.

We introduce the sets $\mathcal{M}_{n}=\left\{(x, \dot{x}, f) \in C(0, T ; V \times H) \times \mathcal{H}^{*} \mid(x, f) \in S_{n}\right\}$ for every $n \in \mathbb{N}$. We have the following upper semicontinuity property.

Corollary 4. If hypotheses of Theorem 2 hold, then $\limsup \mathcal{M}_{n} \subset \mathcal{M}$, where the upper limit is taken in $C(0, T ; V \times H) \times\left(w-\mathcal{H}^{*}\right)$ topology.

\section{Lower semicontinuity property of the solution set}

In order to state a result on lower semicontinuity of the set of mild solutions, we admit the following stronger assumption on the multivalued term.

$\underline{H(F)_{1}}: \quad F, F_{n}:(0, T) \times H \times H \rightarrow \mathcal{P}_{f c}(H)$ are multifunctions satisfying uniformly with respect to $n \in \mathbb{N}$ the conditions

(1) $F(\cdot, u, v)$ is measurable, for all $u, v \in H$,

(2) $F(t, \cdot, \cdot)$ is h-continuous, a.e. $t \in(0, T)$,

(3) $H(F)(3)$ holds

and

$$
\begin{aligned}
& h\left(F_{n}\left(t, u_{1}, v_{1}\right), F\left(t, u_{2}, v_{2}\right)\right) \leq \alpha_{n}(t)\left(\left|u_{1}-u_{2}\right|+\left|v_{1}-v_{2}\right|\right)+\beta_{n}(t) \\
& \text { a.e. } t \in(0, T) \text {, with } \alpha_{n} \in L_{+}^{1}(0, T), \alpha(t)=\sup _{n \in \mathbb{N}} \alpha_{n}(t) \in L_{+}^{1}(0, T) \text { and } \\
& \quad \beta_{n} \rightarrow 0 \text { in } L^{2}(0, T) \text {, as } n \rightarrow+\infty .
\end{aligned}
$$

Remark 1. The estimate (7) holds, for instance, if we suppose that

(a) $h\left(F_{n}\left(t, u_{1}, v_{1}\right), F_{n}\left(t, u_{2}, v_{2}\right)\right) \leq \alpha_{n}(t)\left(\left|u_{1}-u_{2}\right|+\left|v_{1}-v_{2}\right|\right)$ a.e., for every $n \in \mathbb{N}, u_{1}, u_{2}, v_{1}, v_{2} \in H$,

(b) $\quad F_{n}(t, u, v) \rightarrow F(t, u, v)$ in the Hausdorff metric, for all $u, v \in H$, a.e. $t$.

Theorem 3. If hypotheses $H(A)_{1}, H(B)_{1}, H(F)_{1}$ and $\left(H_{0}\right)_{1}$ hold, then $\mathcal{M} \subset$ $\lim \inf \mathcal{M}_{n}$, where the lower limit is taken in $C(0, T ; V \times H) \times\left(s-\mathcal{H}^{*}\right)$ topology. 
Proof. Let $(x, \dot{x}, f) \in \mathcal{M}$. We have to find $\left(x_{n}, \dot{x}_{n}, f_{n}\right) \in \mathcal{M}_{n}$ such that

$$
\begin{gathered}
\left(x_{n}, \dot{x}_{n}\right) \rightarrow(x, \dot{x}) \text { in } C(0, T ; V \times H), \\
f_{n} \rightarrow f \text { in } s-\mathcal{H}^{*} .
\end{gathered}
$$

Define $f_{n}(t, u, v)=\operatorname{proj}\left(f(t), F_{n}(t, u, v)\right)$ for $n \in \mathbb{N}$, where $\operatorname{proj}(a, \mathcal{A})$ denotes the projection of point $a$ onto the set $\mathcal{A}$. Due to Lemma $\alpha$ of [10], we have that $f_{n}$ is measurable in $t$ and continuous in $(u, v)$. Moreover, $f_{n}(t, u, v) \in F_{n}(t, u, v)$ and $H(F)(3)$ implies that $f_{n}$ satisfies the growth condition (5). Therefore applying Corollary 2, we obtain that for every $n \in \mathbb{N}$, the problem

$$
\left\{\begin{array}{l}
\ddot{x}(t)+A_{n}(t, \dot{x}(t))+B_{n} x(t)=\left\{f_{n}(t, x(t), \dot{x}(t))\right\} \text { a.e. } t \in(0, T), \\
x(0)=x_{0}, \quad \dot{x}(0)=x_{1}
\end{array}\right.
$$

possesses a solution $x_{n} \in C(0, T ; V)$ with $\dot{x}_{n} \in \mathcal{W}$. From the equality

$$
\begin{gathered}
\left\langle\ddot{x}_{n}(s)-\ddot{x}(s), \dot{x}_{n}(s)-\dot{x}(s)\right\rangle+\left\langle A_{n}\left(s, \dot{x}_{n}(s)\right)-A(s, \dot{x}(s)), \dot{x}_{n}(s)-\dot{x}(s)\right\rangle+ \\
+\left\langle B_{n} x_{n}(s)-B x(s), \dot{x}_{n}(s)-\dot{x}(s)\right\rangle=\left\langle f_{n}\left(s, x_{n}(s), \dot{x}_{n}(s)\right)-f(s), \dot{x}_{n}(s)-\dot{x}(s)\right\rangle
\end{gathered}
$$

a.e. $s \in(0, T)$, by integrating by parts, using $H(A)_{1}, H(B)_{1}$, similarly as in the proof of Proposition 2, we obtain

$$
\begin{gathered}
\left|\dot{x}_{n}(t)-\dot{x}(t)\right|^{2}+m\left\|x_{n}(t)-x(t)\right\|^{2} \leq \sigma_{n}+ \\
+2 \int_{0}^{t}\left|f_{n}\left(s, x_{n}(s), \dot{x}_{n}(s)\right)-f(s) \| \dot{x}_{n}(s)-\dot{x}(s)\right| d s
\end{gathered}
$$

for all $t \in[0, T]$, where $\sigma_{n}=2\left\|\widehat{A}_{n}(\dot{x})-\widehat{A}(\dot{x})\right\|_{\mathcal{V}^{*}}\left\|\dot{x}_{n}-\dot{x}\right\|_{\mathcal{V}}+C\left\|B_{n}-B\right\|\left\|x_{n}\right\|_{\mathcal{V}}$ $\left\|\dot{x}_{n}-\dot{x}\right\|_{\mathcal{V}}$ and $C>0$. Taking into account that

$$
\begin{gathered}
\left|f_{n}\left(s, x_{n}(s), \dot{x}_{n}(s)\right)-f(s)\right|=d\left(f(s), F_{n}\left(s, x_{n}(s), \dot{x}_{n}(s)\right)\right) \leq \\
\leq h\left(F(s, x(s), \dot{x}(s)), F_{n}\left(s, x_{n}(s), \dot{x}_{n}(s)\right)\right) \leq \\
\leq \alpha_{n}(s)\left(\left|x_{n}(s)-x(s)\right|+\left|\dot{x}_{n}(s)-\dot{x}(s)\right|\right)+\beta_{n}(s) \text { a.e. } s \in(0, T),
\end{gathered}
$$

we have

$$
\begin{aligned}
& \left|\dot{x}_{n}(t)-\dot{x}(t)\right|^{2}+m\left\|x_{n}(t)-x(t)\right\|^{2} \leq \sigma_{n}+2 \int_{0}^{t} \alpha(s)\left|\dot{x}_{n}(s)-\dot{x}(s)\right|^{2} d s+ \\
& +2 \int_{0}^{t} \alpha(s)\left|x_{n}(s)-x(s)\right|\left|\dot{x}_{n}(s)-\dot{x}(s)\right|^{2} d s+2 \int_{0}^{t} \beta_{n}(s)\left|\dot{x}_{n}(s)-\dot{x}(s)\right| d s
\end{aligned}
$$

for all $t \in[0, T]$. Applying the inequality $2 a b \leq a^{2}+b^{2}, a, b>0$ to the last two integrals on the right hand side and using the fact that $|\cdot| \leq \gamma\|\cdot\|$ with $\gamma>0$, we have

$$
\left|\dot{x}_{n}(t)-\dot{x}(t)\right|^{2}+m\left\|x_{n}(t)-x(t)\right\|^{2} \leq \sigma_{n}+\left\|\beta_{n}\right\|_{L^{2}(0, T)}^{2}+
$$




$$
+\int_{0}^{t}\left[(3 \alpha(s)+1)\left|\dot{x}_{n}(s)-\dot{x}(s)\right|^{2}+\alpha(s) \gamma^{2}\left\|x_{n}(s)-x(s)\right\|^{2}\right] d s
$$

for all $t \in[0, T]$. Invoking the Gronwall inequality, we get

$$
\left|\dot{x}_{n}(t)-\dot{x}(t)\right|^{2}+\left\|x_{n}(t)-x(t)\right\|^{2} \leq C\left(\sigma_{n}+\left\|\beta_{n}\right\|_{L^{2}}^{2}\right) \text { for all } t \in(0, T),
$$

where $C$ is a positive constant independent of $n$. From Lemma $1, H(A)_{1}$ and $H(B)_{1}$, we infer that $\lim \sigma_{n}=0$. Hence, we have shown (8).

In order to prove (9), by using (10), we write

$$
\begin{gathered}
\int_{0}^{T}\left|f_{n}\left(s, x_{n}(s), \dot{x}_{n}(s)\right)-f(s)\right|^{q} d s \leq \\
\leq 2^{q-1} \int_{0}^{T}(\alpha(s))^{q}\left(\left|x_{n}(s)-x(s)\right|+\left|\dot{x}_{n}(s)-\dot{x}(s)\right|\right)^{q} d s+2^{q-1}\left\|\beta_{n}\right\|_{L^{2}}^{2} .
\end{gathered}
$$

In view of (8) and the convergence $\beta_{n} \rightarrow 0$ in $L^{2}(0, T)$, we easily get (9). This completes the proof.

Corollary 5. If hypotheses of Theorem 3 hold, then $\mathcal{M}_{n} \stackrel{K}{\longrightarrow} \mathcal{M}$ in $C(0, T ; V \times$ $H) \times\left(s-\mathcal{H}^{*}\right)$ topology. This follows from Theorem 3 and the fact that Corollary 4 implies $\lim \sup \mathcal{M}_{n} \subset \mathcal{M}$ in this topology.

\section{References}

1. N. U. Ahmed and S. Kerbal, Optimal control of nonlinear second order evolution equations, J. Appl. Math. Stochastic Anal. 6 (1993), 123-136.

2. J.-P. Aubin and A. Cellina, Differential Inclusions, Springer-Verlag, New York (1984).

3. Z. Denkowski, S. Migórski and N.S. Papageorgiou, An Introduction to Nonlinear Analysis: Theory. Kluwer Academic/Plenum Publishers, New York (2003).

4. Z. Denkowski and S. Mortola, Asymptotic behavior of optimal solutions to control problems for systems described by differential inclusions corresponding to partial differential equations, J. Optimiz. Theory Appl. 78 (1993), 365-391.

5. J. L. Lions, Optimal Control of Systems Governed by Partial Differential Equations, Springer-Verlag (1971).

6. S. Migórski, Variational stability analysis of optimal control problems for systems governed by nonlinear second order evolution equations, J. Math. Systems, Estim. Control 6 (1996), 1-24.

7. S. Migórski, Control problems for systems described by nonlinear second order evolution inclusions, Nonlinear Anal. Theory Meth. Appl. 30 (1997), 419-428.

8. S. Migórski, Existence of solutions to nonlinear second order evolution inclusions without and with impulses, Dynamics Cont. Discr. Impul. Syst. Series B, 18 (2011), 493-520.

9. S. Migórski, A. Ochal and M. Sofonea, Nonlinear Inclusions and Hemivariational Inequalities. Models and Analysis of Contact Problems, Advances in Mechanics and Mathematics 26, Springer, New York (2013).

10. N. S. Papageorgiou, Continuous dependence results for a class of evolution inclusions, Proc. Edinburgh Math. Soc. 35 (1992), 139-158.

11. E. Zeidler, Nonlinear Functional Analysis and its Applications, II, Springer-Verlag, New York (1990). 